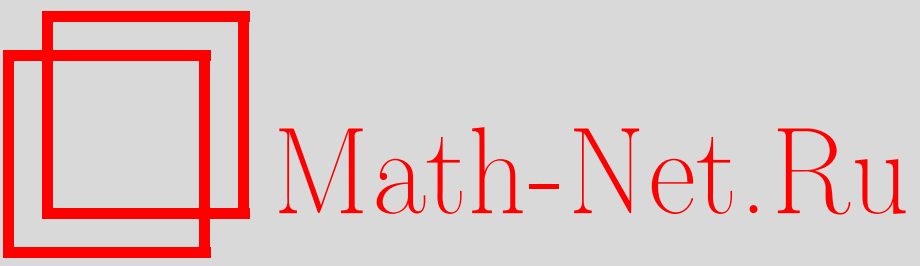

Нгуен Мань Хунг, О гладкости решения задачи Дирихле для гиперболических систем в областях с негладкой границей, УМН, 1998, том 53, выпуск 2, 157-158

DOI: https://doi.org/10.4213/rm42

Использование Общероссийского математического портала Math-Net.Ru подразумевает, что вы прочитали и согласны с пользовательским соглашением

http://www.mathnet.ru/rus/agreement

Параметры загрузки:

IP: 35.174 .16 .151

26 апреля 2023 г., 15:40:35 


\title{
О ГЛАДКОСТИ РЕШЕНИЯ ЗАДАЧИ ДИРИХЛЕ ДЛЯ ГИПЕРБОЛИЧЕСКИХ СИСТЕМ В ОБЛАСТЯХ С НЕГЛАДКОЙ ГРАНИЦЕЙ
}

\author{
НГУЕН МАНЬ ХУНГ
}

Эллиптические краевые задачи в областях с негладкой границей были рассмотрены в работах В. А. Кондратьева [3], [4]. Им были установлены условия нетеровости и теоремы о гладкости решения. Аналогичные вопросы для эллиптической системы рассмотрели В. Г. Мазья, Б. А. Пламеневский в работах [1], [2]. Краевые задачи для нестационарных систем в областях с гладкой границей рассматривались О. А. Ладыженской в [5].

Рассмотрим следующую систему:

$$
(-1)^{m-1}\left[\sum_{|p|,|q|=1}^{m} D^{p} a_{p q}(x, t) D^{q} u+\sum_{|p|=1}^{m} a_{p}(x, t) D^{p} u+a(x, t) u\right]-u_{t t}=f,
$$

где $D^{\alpha}=\partial^{|\alpha|} / \partial x_{1}^{\alpha_{1}} \cdots \partial x_{n}^{\alpha_{n}}, \alpha=\left(\alpha_{1}, \ldots, \alpha_{n}\right), u_{t^{k}}=\partial^{k} u / \partial t^{k}, a_{p q}, a_{p}, a-$ бесконечно дифоференцируемые комплексные матрицы размера $r \times r$ в $\bar{Q}_{T}=\bar{\Omega} \times[0, T], \Omega \subset \mathbb{R}^{n}$ - ограниченная область, граница которой $\partial \Omega$ является кусочно-гладкой поверхностью, состоящей из конечного числа $(n-1)$-мерных гладких поверхностей $\Gamma_{i}$. Предполагается, что поверхность $\Gamma_{i}$ может пересекаться только с поверхностями $\Gamma_{i-1}, \Gamma_{i+1}$ вдоль гладких $(n-2)$-мерных многообразий $l_{i-1}, l_{i+1}$. Мы подробно остановимся на случае, когда $\partial \Omega$ состоит из двух поверхностей $\Gamma_{1}, \Gamma_{2}$, пересекающихся вдоль многообразия $l_{0}$. Аналогично рассматривается поведение решений около линии пересечения остальных многообразий.

Предположим, что в окрестности каждой точки из $l_{0}$ множество $\bar{\Omega}$ дифффеоморфно двугранному углу. Для каждой точки $P \in l_{0}$ определены два касательных к $\Omega$ полупространства $T_{1}(P)$, $T_{2}(P)$ и двумерная плоскость $\pi(P)$, нормальная к $l_{0}$. Обозначим через $\gamma(P)$ угол в плоскости $\pi(P)$ (со стороны $\Omega$ ), ограниченный лучами $T_{1} \cap \pi(P), T_{2} \cap \pi(P)$, через $\beta(P)$ - раствор этого угла. Введем в рассмотрение некоторые функциональные пространства:

$H_{m}(\Omega)$ - гильбертово пространство комплексных $r$-мерных вектор-функций, имеющих внутри $\Omega$ обобщенные производные до порядка $m$ включительно, таких, что

$$
\|u\|_{m, \Omega}^{2}=\sum_{0 \leqslant|p| \leqslant m} \int_{\Omega}\left|D^{p} u\right|^{2} d x<+\infty
$$

$\stackrel{\circ}{H}_{m}(\Omega)$-подпространство $H_{m}(\Omega)$, плотньц множеством в котором являются бесконечно дифоференцируемые комплексные $r$-мерные вектор-функции с компактным носителем в $\Omega$;

$H_{m}\left(Q_{T}\right)$ - пространство комплексных $r$-мерных вектор-фуннкций с нормой

$$
\|u\|_{m, Q_{T}}^{2}=\sum_{0 \leqslant|p|+k \leqslant m} \int_{Q_{T}}\left|D^{p} u_{t^{k}}\right|^{2} d x d t
$$

$V_{m}\left(Q_{T}\right)$ - пространство комплексных $r$-мерных вектор-функций с нормой

$$
\|u\|_{\rho, m, Q_{T}}^{2}=\sum_{0 \leqslant|p|+k \leqslant m} \int_{Q_{T}} \rho^{2(|p|-m)}\left|D^{p} u_{t^{k}}\right|^{2} d x d t
$$

где $\rho(x)$ - функция, бесконечно дифференцируемая и положительная всюду, кроме $l_{0}$, и совпадающая в некоторой окрестности $l_{0} \mathrm{c} r\left(x, l_{0}\right)$ - расстоянием от $x$ до $l_{0}$.

$L(x, t, D)=\sum_{|p|,|q|=1}^{m} D^{p} a_{p q}(x, t) D^{q}+\sum_{|p|=1}^{m} a_{p}(x, t) D^{p}+a(x, t), \quad D_{\nu}^{j}=\frac{\partial^{j}}{\partial \nu^{j}}(j=1, \ldots, m-1)$, где $\nu$-нормаль к $S_{T}=\partial \Omega \times[0, T]$, направленная вне $Q_{T}, a_{p q}$ удовлетворяют условию

$$
\sum_{|p|,|q|=m}(-1)^{|p|} \int_{\Omega} a_{p q}(x, t) D^{q} u \overline{D^{p} u} d x \geqslant \mu_{0}\|u(x, t)\|_{m, \Omega}
$$


для любого $u \in \stackrel{\circ}{H}(\Omega)$ при почти всех $t \in[0, T], \mu_{0}=$ const $>0$.

$$
\begin{gathered}
(-1)^{m-1} L(x, t ; D) u-u_{t t}=f, \\
\left.u\right|_{t=0}=0,\left.\quad u_{t}\right|_{t=0}=0, \\
\left.D_{\nu}^{j} u\right|_{S_{T}}=0 \quad(j=1, \ldots, m-1) .
\end{gathered}
$$

Пусть $t \in[0, T]$. Для $S_{0}=l_{0} \times[0, T]$ обозначим сечение $S_{0}$ гиперплоскостью $\{t=$ const $\}$ через $S_{0}^{t}$, а через $\left\{U_{t}^{\alpha}\right\}_{\alpha}$ обозначим достаточно мелкое покрытие $\bar{\Omega}_{t}$ координатными окрестностями. Если $U_{t}^{\alpha}$ пересекается с $S_{0}^{t}$, то каждой точке $(x, t) \in U_{t}^{\alpha}$ сопоставим координаты $(y, z, t)$, где $(z, t)$ - точка из $S_{0}^{t}$, ближайшая к точке $(x, t)$, а $y=\left(y_{1}, y_{2}\right)$ - координаты точки $(x, t)$ в плоскости $\pi(z, t)$.

Запишем оператор $L$ в точке $(z, t)$ в виде $L\left(z, t ; D_{y}, D_{z}\right)$. Обозначим через $(r, \omega)$ полярные координаты точки $y$, через $L_{0}$ - главную часть полинома $L(z, t ; \eta, \xi)$ (по переменным $\eta, \xi$ ) и положим

$$
L_{0}\left(z, t ; D_{y}, 0\right)=r^{-2 m} Q\left(z, t ; \omega, D_{\omega} ; r D_{r}\right) \quad\left(D_{r}=i^{-1} \partial / \partial r\right) .
$$

Обозначим через $\lambda(z, t)$ собственные числа задачи

$$
\begin{array}{cl}
Q\left(z, t ; \omega, D_{\omega} ; \lambda\right) v(\omega)=0, & 2|\omega|<\beta(z, t), \\
D_{\omega}^{j} v(\omega)=0, \quad 2 \omega= \pm \beta(z, t) & (j=0, \ldots, m-1) .
\end{array}
$$

TEOPEMA 1. Пусть $f_{t^{k}} \in L_{2}\left(Q_{T}\right) n p u k \leqslant 2 m-1, f_{t^{k}}(x, 0) \in \stackrel{\circ}{H}_{m}(\Omega) \cap H_{2 m(m-1)}(\Omega) n p u$ $k \leqslant 2(m-1)$, и пусть при всех $(z, t) \in S_{0}$ в полосе $1-2 m \leqslant \operatorname{Im} \lambda \leqslant 1-m$ нет собственных чисел задачи (5)-(6). Тогда обобщенное решение задачи (2)-(4) принадлежит $H_{2 m}\left(Q_{T}\right)$ $u$

$$
\|u\|_{2 m, Q_{T}} \leqslant C \sum_{k=0}^{2 m-1}\left\|f_{t^{k}}\right\|_{L_{2}\left(Q_{T}\right)}, \quad C=\text { const }>0 .
$$

ДоКАЗАТЕЛЬСТво. Перепишем систему (1) в виде

$$
L(x, t ; D) u=(-1)^{m-1}\left(u_{t t}-f\right) .
$$

Из теоремы 2.2 работы [6] следует, что при почти всех $t \in[0, T]$ правая часть системы (7) принадлежит $L_{2}\left(\Omega_{t}\right)$. Утверждение теоремы получается методом индукции.

TEOPEMA 2. Ecлu $f_{t^{k}} \in V_{h}\left(Q_{T}\right) n p u k \leqslant 2 m-1, f_{t^{k}}(x, 0) \in \stackrel{\circ}{H}_{m}(\Omega) \cap H_{m(2 m-1)}(\Omega)$ при $k \leqslant 2 m-2$, а в полосе $1-2 m-h \leqslant \operatorname{Im} \lambda \leqslant 1-m$ нет собственных чисел задачи (5)-(6) при всех $(z, t) \in S_{0}$, то обобщенное решение $u(x, t)$ задачи (2)-(4) принадлежит $V_{2 m+h}\left(Q_{T}\right)$ и справедливо неравенство

$$
\|u\|_{\rho, 2 m+h, Q_{T}} \leqslant C \sum_{k=0}^{2 m-1}\left\|f_{t^{k}}\right\|_{\rho, h, Q_{T}}, \quad C=\text { const }>0 .
$$

Выражаю глубокую благодарность профессору В.А. Кондратьеву за помощь в работе.

\section{СПИСОК ЛИТЕРАТУРЫ}

[1] Мазья В.Г., Пламеневский Б. А. // Труды ММО. 1978. Т. 37. С. 49-93. [2] Мазья В. Г., Пламеневский Б. А. // Проблемы матем. анализа, ЛГУ. 1977. №6. С. 85-145. [3] Кондратьев В. А. // Труды ММО. 1967. Т. 16. С. 209-292. [4] Кондратьев В. А. // Дифференц. уравнения. 1970. Т. 6. № 10. С. 1831-1843. [5] Ладыженская О. А. // Матем. сб. 1958. Т. 45 (87). № 2. С. 123-158. [6] Нгуен Мань Хунг // УМН. 1997. Т. 52. №6. С. 189-190. 